\title{
Explicit renormalization of the nucleon-nucleon interaction in chiral EFT with a finite cutoff.
}

\section{A. M. Gasparyan ${ }^{a, b, *}$ and E. Epelbaum ${ }^{a}$}

${ }^{a}$ Ruhr-Universität Bochum, Fakultät für Physik und Astronomie, Institut für Theoretische Physik II, D-44780 Bochum, Germany

${ }^{b}$ NRC “Kurchatov Institute” - ITEP, B. Cheremushkinskaya 25, 117218 Moscow, Russia

E-mail: ashot.gasparyan@rub.de, evgeny.epelbaum@rub.de

Nucleon-nucleon interaction is studied within chiral effective field theory with a finite cutoff at next-to-leading order in the chiral expansion. The leading order interaction is resummed in a nonperturbative manner, whereas the next-to-leading-order terms are treated perturbatively. Some aspects of renormalizability of such a scheme are addressed. In particular, it is analyzed whether the power-counting breaking terms originating from the integration regions with momenta of the order of the cutoff can be absorbed by the renormalization of the low energy constants corresponding to the leading contact interactions.

*** Particles and Nuclei International Conference - PANIC2021 ***

*** 5 - 10 September, $2021 * * *$

*** Online ***

${ }^{*}$ Speaker 


\section{Introduction}

In recent years, chiral effective field theory (chiral EFT) has been widely used for studying two-nucleon dynamics, few-nucleon and many-nucleon systems.

One of the most efficient schemes within chiral EFT is the approach suggested by Weinberg $[1,2]$. It consists in applying the chiral power counting to the effective potential and iterating it non-perturbatively introducing a regulator in the form of a finite cutoff $\Lambda$ of the order of the chiral symmetry breaking scale. This approach is phenomenologically quite successful, see, e.g., Refs. [3-5] for recent applications and Ref. [6] for a review. From a more fundamental point of view, there still remain open questions such as explicit renormalizability of this theory.

The issue of the renormalization of the nucleon-nucleon $(\mathrm{NN})$ interaction is subject to hot debate, see Refs. [7-10] for recent discussions. In Ref. [16], the renormalizability of the NN EFT with a finite cutoff was analyzed at next-to-leading (NLO) order in the chiral expansion for the perturbative leading order (LO) interaction summed to an arbitrary order.

In this talk, we report on a further development along this line by considering the nonperturbative effects.

\section{Perturbative renormalization at NLO}

Our scheme is based on the chiral effective Lagrangian:

$$
\mathcal{L}_{\text {eff }}=\mathcal{L}_{\pi}^{(2)}+\mathcal{L}_{\pi N}^{(1)}+\mathcal{L}_{N N}^{(0)}+\mathcal{L}_{N N}^{(2)}+\ldots
$$

organized as an expansion in terms of the number of derivatives and the power of the pion mass $M_{\pi}$. This corresponds to an expansion of observables in terms of the ratio of the soft and the hard scale $Q=\frac{q}{\Lambda_{b}}$, where the soft scale is given by external on-shell nucleon momenta $p_{\text {on }}$, and $M_{\pi}$ and the hard chiral symmetry breaking scale $\Lambda_{b}$ can be associated with the $\rho$-meson mass.

In Weinberg's approach, the chiral order of potential ( $2 \mathrm{~N}$-irreducible) contributions is given by the power counting formula

$$
D=2 L+\sum_{i}\left(d_{i}+\frac{n_{i}}{2}-2\right)
$$

where $L$ is the number of loops, all vertices of the diagram, $n_{i}$ is the number of nucleon lines in vertex $i$ and $d_{i}$ is the number of derivatives and the pion-mass insertions at vertex $i$. From this formula, it follows that the LO potential consists of the derivativeless contact interaction and the one-pion exchange contribution. The NLO potential contains two-pion-exchange contributions and subleading contact terms. In practice, one can promote certain terms to leading order by numerical arguments.

$2 \mathrm{~N}$-reducible graphs are enhanced due to the infrared singularity of the nucleon propagators. In particular, all iterations of the LO potential $V_{0}$ have to be taken into account at the same order. 
The LO and NLO amplitudes are given then by the series (in case they converge)

$$
\begin{aligned}
& T_{0}=V_{0}+V_{0} G V_{0}+V_{0} G V_{0} G V_{0}+\cdots=\sum_{n=0}^{\infty} T_{0}^{[n]}, \\
& T_{2}=V_{2}+V_{2} G V_{0}+V_{0} G V_{2}+V_{2} G V_{0} G V_{0}+\cdots=\sum_{m, n=0}^{\infty} T_{2}^{[m, n]}
\end{aligned}
$$

These series contain multiple integrals in the momentum space that converge at momenta $p \sim \Lambda$ (without a regulator they would diverge). For the LO amplitude, this means that all terms in Eq. (3) are of order $\left(\frac{\Lambda}{\Lambda_{b}}\right)^{n} \sim O\left(Q^{0}\right)$, with $n=0,1,2 \ldots$, and the inverse powers of $\Lambda_{b}$ come from the LO potential. This is consistent with Weinberg power counting. A formal proof of this statement was given in Ref. [16].

However, for the NLO amplitude, the integration regions with $p \sim \Lambda$ produce power counting breaking terms of order $O\left(Q^{0}\right)$ (instead of $O\left(Q^{2}\right)$ ). We call a theory renormalizable in the sense of EFT if such terms can be absorbed by a redefinition (renormalization) of the low energy constants (LECs) of a lower order (in this case, order $Q^{0}$ ). In Ref. [16], it was shown that in case the series in Eq. (4) converges, such a theory is indeed renormalizable under rather general conditions. In particular, one can construct the renormalized amplitude $\mathbb{R}\left(T_{2}^{[m, n]}\right)$ for each term in Eq. (4), that satisfies the inequality

$$
\left|\mathbb{R}\left(T_{2}^{[m, n]}\right)\left(p_{\text {on }}\right)\right| \leq \frac{8 \pi^{2} \mathcal{M}_{1}}{m_{N} \Lambda_{b}}\left(\mathcal{M}_{2} \frac{\Lambda}{\Lambda_{b}}\right)^{m+n} \frac{p_{\text {on }}^{2}}{\Lambda_{b}^{2}} \log \Lambda / M_{\pi},
$$

where $\mathcal{M}_{1}, \mathcal{M}_{2}$ are some constants of order 1 .

The situation when the series in Eq. (4) has to be resummed non-perturbatively is, however, more complicated.

\section{Non-perturbative renormalization at NLO}

For the LO amplitude the non-perturbative analysis is similar to the perturbative case. It is convenient to apply the Fredholm method and represent the LO amplitude as

$$
T_{0}=V_{0} R=\bar{R} V_{0}, \quad R=\frac{1}{\mathbb{1}-G V_{0}}=\frac{N}{D}, \quad \bar{R}=\frac{1}{\mathbb{1}-V_{0} G}=\frac{\bar{N}}{D},
$$

where the minor $N$ and the Fredholm determinant $D$ can be expanded in convergent series in $V_{0}$ :

$$
N=\sum_{i=0}^{\infty} N_{i}, \quad D=\sum_{i=0}^{\infty} D_{i}
$$

which makes it possible to connect the non-perturbative case to the perturbative expansion and derive the power counting for the LO amplitude. The non-perturbative dynamics is now contained in the Fredholm determinant, which is a number that depends only on the on-shell momentum (energy): $D=D\left(p_{\text {on }}\right)$. 
For the NLO amplitude, it is possible to sum the series for the renormalized subtracted quantities $\mathbb{R}\left(T_{2}^{[m, n]}\right)$ in a closed form:

$$
\mathbb{R}\left(T_{2}\right)\left(p_{\text {on }}\right)=\sum_{m, n=0}^{\infty} \mathbb{R}\left(T_{2}^{[m, n]}\right)\left(p_{\text {on }}\right)=T_{2}\left(p_{\text {on }}\right)-T_{2}\left(p_{\text {on }}=0\right)\left[\frac{\psi_{p_{\text {on }}}(0)}{\psi_{p_{\text {on }}=0}(0)}\right]^{2}
$$

where $\psi_{p_{\text {on }}}(0)$ is the LO scattering wave function at the origin, which corresponds to the subtraction at $p_{\text {on }}=0: \mathbb{R}\left(T_{2}\right)\left(p_{\text {on }}=0\right)=0$. Introducing the quantities $N_{\psi}$ and $N_{2}$ via

$$
\psi_{p_{\text {on }}}(0)=\frac{N_{\psi}\left(p_{\text {on }}\right)}{D\left(p_{\text {on }}\right)}, \quad T_{2}\left(p_{\text {on }}\right)=\frac{N_{2}\left(p_{\text {on }}\right)}{D\left(p_{\text {on }}\right)^{2}}
$$

we can rewrite Eq. (8) as

$$
\mathbb{R}\left(T_{2}\right)\left(p_{\text {on }}\right)=\frac{1}{D\left(p_{\text {on }}\right)^{2}}\left[N_{2}\left(p_{\text {on }}\right)-N_{2}(0) \frac{N_{\psi}\left(p_{\text {on }}\right)}{N_{\psi}(0)}\right] .
$$

Both $N_{\psi}$ and $N_{2}$ can be represented as convergent series in $V_{0}$, which allows one to derive the correct power counting for the NLO amplitude. However, in contrast to the perturbative case, an additional condition has to be satisfied by the LO potential to guarantee the renormalizability, namely

$$
N_{\psi}(0) \neq 0
$$

when $V_{0}$ is rescaled as $V_{0} \rightarrow \lambda V_{0}$, for any complex $|\lambda|<1$.

\section{Summary}

We have analyzed the renormalizability of the nucleon-nucleon effective field theory with a finite cutoff at next-to-leading order paying a particular attention to non-perturbative effects. We have shown that the next-to-leading amplitude satisfies the expected power counting if one imposes a certain constraint on the leading order potential.

\section{Acknowledgments}

This research was supported by DFG (Grant No. 426661267), by DFG and NSFC through funds provided to the Sino-German CRC 110 "Symmetries and the Emergence of Structure in QCD” (NSFC Grant No. 12070131001, Project-ID 196253076 - TRR 110) and by BMBF (Grant No. 05P18PCFP1).

\section{References}

[1] S. Weinberg, Phys. Lett. B251, 288 (1990)

[2] S. Weinberg, Nucl. Phys. B363, 3 (1991)

[3] P. Reinert, H. Krebs, E. Epelbaum, Eur. Phys. J. A54, 86 (2018), 1711.08821

[4] D.R. Entem, R. Machleidt, Y. Nosyk, Phys. Rev. C96, 024004 (2017), 1703.05454 
[5] P. Reinert, H. Krebs, E. Epelbaum, Phys. Rev. Lett. 126, 092501 (2021), 2006. 15360

[6] E. Epelbaum, H. Krebs, P. Reinert, Front. in Phys. 8, 98 (2020), 1911. 11875

[7] E. Epelbaum, J. Gegelia, U.G. Meißner, Nucl. Phys. B 925, 161 (2017), 1705. 02524

[8] E. Epelbaum, A.M. Gasparyan, J. Gegelia, U.G. Meißner, Eur. Phys. J. A54, 186 (2018), 1810.02646

[9] U. van Kolck, Front. in Phys. 8, 79 (2020), 2003.06721

[10] E. Epelbaum, A.M. Gasparyan, J. Gegelia, U.G. Meißner, X.L. Ren, Eur. Phys. J. A 56, 152 (2020), 2001.07040

[11] P.F. Bedaque, U. van Kolck, Ann. Rev. Nucl. Part. Sci. 52, 339 (2002), nucl-th/0203055

[12] E. Epelbaum, H.W. Hammer, U.G. Meißner, Rev.Mod.Phys. 81, 1773 (2009), 0811.1338

[13] R. Machleidt, D. Entem, Phys.Rept. 503, 1 (2011), 1105.2919

[14] E. Epelbaum, U.G. Meißner, Ann. Rev. Nucl. Part. Sci. 62, 159 (2012), 1201.2136

[15] H.W. Hammer, S. König, U. van Kolck, Rev. Mod. Phys. 92, 025004 (2020), 1906.12122

[16] A.M. Gasparyan, E. Epelbaum (2021), 2110.15302 